\title{
Syndrome de défaillance multiviscérale
}

\section{Piet van Spijk}

Dr méd., dr en philosophie, spécialiste en médecine interne, président du Forum Médecine et philosophie

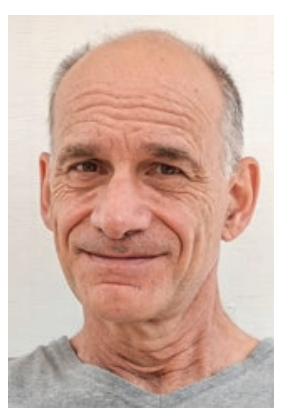

Chères lectrices, chers lecteurs, j'ai tout à fait conscience que le présent article risque de provoquer un malaise. Je comprendrais que vous renonciez à le lire car certaines choses sont difficiles à entendre, notamment le diagnostic d'une mort prochaine. Ce type d'informations peut provoquer des sentiments de peur tellement forts qu'ils nous submergent et nous rendent la vie difficile.

Pour toutes les personnes motivées à poursuivre, je propose une brève histoire en guise d'introduction à la thématique. Une jeune maman part au travail un matin en laissant à son mari le soin de s'occuper de Lisa, leur fille de sept ans. Vers midi, la petite dit à son père qu'elle est fatiguée et qu'elle va se coucher. Le papa qui trouve ce comportement inhabituel de la part de sa fille lui apporte un verre d'eau et prend sa température: $37,2^{\circ}$. Une demi-heure plus tard, Lisa se met à vomir et sa température grimpe à $38,4^{\circ}$. Inquiet, le papa appelle le médecin, puis tout s'enchaîne très vite. Visite d'urgence au cabinet, ambulance, admission à l'hôpital universitaire le plus proche, soins intensifs. La maman est avertie, mais quand elle arrive à l'hôpital, il est déjà trop tard. Diagnostic: syndrome de défaillance multiviscérale suite à une septicémie méningococcique.

Jeune médecin assistant à l'époque, je n'ai pas été directement confronté à cette tragédie. Elle m’a néanmoins profondément marquée, car elle m’a fait comprendre que lorsque des substances toxiques ou d'autres pathogènes produisent des dégâts importants dans des systèmes aussi complexes que le corps humain, un système de régulation défaillant peut entraîner la destruction d'un autre système qui en dépend et ainsi de suite. Le fait de savoir que ces enchaînements peuvent être très rapides m'a souvent aidé à éviter des catastrophes dans ma pratique clinique. Jusque-là, on s'en sort plutôt mal. Et je ne suis pas le seul à avoir eu ce type d'expérience, c'est aussi arrivé à bon nombre de mes collègues. Sensibilisé par la pandémie en cours, je me suis souvent demandé ce que pensaient les méningocoques lorsque, bien implantés dans l'hôte qui leur permet de survivre, ils courent à la mort. J'en suis arrivé à la conclusion limpide qu'ils ne pensent pas, les bactéries étant incapables de penser.
Et pourtant, j'ai repensé récemment à la tragédie de Lisa et à la situation dans laquelle nous nous trouvons et des pensées pleines d'inquiétude m'ont assaillies ne me laissant plus de répit. D’une part, il y a la pensée que les métabolismes complexes et complémentaires régissant les organismes humains ressemblent à bien des points à ceux de l'écosystème planétaire. A cette réflexion, j'ai fait le lien avec la maladie de Lisa et comparé le réchauffement de la planète d'un degré à une légère montée de fièvre. Mais que se passerait-il si les températures augmentaient de deux, voire trois degrés en moyenne, et pas seulement dans les Alpes et l'Arctique, et que cela génère d'immenses feux dans la toundra arctique produisant une énorme quantité de $\mathrm{CO}_{2}$ ?

Serait-ce le signe que l'être humain, sorte de «méningocoque de l'écosystème», est en train de créer un syndrome de défaillance multiviscérale de cet organisme complexe qu'est la planète? Que se passerait-il si le réchauffement mondial d'un degré (et de deux à trois degrés dans les régions exposées) correspondait au moment où Lisa se met à vomir et qu'il est déjà trop tard? Que se passerait-il si les actions prévues dans 10, 20, 30 ou 50 ans arrivaient beaucoup trop tard étant donné que l'écosystème de la planète est d'ores et déjà irréversiblement perturbé et que la survie de l'être humain y est compromise?

Chères lectrices et chers lecteurs, j'espère que vous saurez trouver des arguments convaincants pour apaiser mes craintes. Il est possible que ma comparaison ne tienne pas la route. Bien sûr que le réchauffement climatique existe, mais il n'est sans doute pas comparable à un syndrome de défaillance multiviscérale de l'écosystème planétaire. Si c'est le cas, il suffit d'initier tranquillement le traitement. Il se peut que les feux dans l'Arctique et en Méditerranée, les records de chaleur au Canada et les inondations en Allemagne ne soient que des coïncidences qui ne doivent pas particulièrement nous alerter. J'espère vraiment que vous saurez m'en convaincre!

P.S.: Si vous n'êtes pas en mesure d'apaiser mes craintes ce que je n'espère pas -, que diable faut-il faire? 